\title{
Contents, Vol. 195, Supplement 1, 1997
}

1 Oral Isotretinoin. Where Now, Where Next? Saurat, J.-H. (Geneva)

4 Does Oral Isotretinoin Prevent Propionibacterium acnes Resistance?

Coates, P.; Adams, C.A.; Cunliffe, W.J.; McGinley, K.T.; Eady, E.A.; Leyden, J.J.; Ravenscroft, J.; Vyakrnam, S.; Vowels, B. (Leeds/Philadelphia, Pa.)

10 How Cost-Effective Is Oral Isotretinoin?

Newton, J.N. (Oxford)

15 Scarred for Life?

Layton, A.M.; Seukeran, D.; Cunliffe, W.J. (Leeds)

22 How Safe Is Oral Isotretinoin?

Meigel, W.N. (Hamburg)

29 Oral Isotretinoin. How Can We Treat Difficult Acne Patients?

Leyden, J.J. (Philadelphia, Pa.)

34 Oral Isotretinoin Treatment Policy. Do We All Agree?

Ortonne, J.-P. (Nice)

38 Discussion. Questions and Answers

KAKG E \\ (C) 1997 S. Karger AG, Basel III

E-Mail karger@karger.ch The list of contents is available at:

Fax+41 613061234 http://www.karger.ch/joumals/der/dercont.htm

http://www.karger.ch 